\title{
Process fairness, outcome fairness, and dynamic consistency: Experimental evidence for risk and ambiguity
}

\author{
Stefan T. Trautmann ${ }^{1}$ • Gijs van de Kuilen ${ }^{2}$
}

Published online: 4 February 2017

(C) The Author(s) 2017. This article is published with open access at Springerlink.com

\begin{abstract}
Literature on fairness preferences distinguishes between outcome fairness, concerning the final allocation of payoffs, and process fairness, concerning the expected allocation of payoffs. It is not obvious, however, whether process fairness can consistently be implemented. Once uncertainty is resolved and outcomes are determined, the ex-ante procedurally fair decision maker may become consequentialist expost, and reconsider her choice on the basis of the observed outcomes. We present experimental evidence on dynamic consistency of social preferences under both known risk and ambiguity. A significant share of people subscribe to process fairness both before and after the resolution of uncertainty.
\end{abstract}

Keywords Procedural fairness · Distributional fairness · Process fairness · Outcome fairness · Dynamic consistency

JEL Classifications C91 $\cdot \mathrm{D} 63 \cdot \mathrm{D} 81$

\section{Introduction}

Preferences that are affected by considerations of fairness have been widely used to explain contractual agreements in organizations and firms. The literature distinguishes

Stefan T. Trautmann

trautmann@uni-hd.de

Gijs van de Kuilen

g.v.d.kuilen@uvt.nl

1 Alfred-Weber-Institute for Economics, University of Heidelberg, Bergheimer Str. 58 (Room 01.029), 69115 Heidelberg, Germany

2 Department of Economics \& CentER, Tilburg University, P.O. Box 90153, 5000LE Tilburg, The Netherlands 
between preferences for outcome fairness, where the agent is concerned about the actual distribution of payoffs, and preferences for process fairness, where the agent is concerned about the random process by which outcomes are created, but not what these outcomes actually are (e.g., Trautmann and Vieider 2012). That is, outcome fairness is consequentialist while process fairness is non-consequentialist: an agent with process fairness preferences takes into consideration the counterfactual outcomes at branches of the decision tree that never materialize. A potential problem of non-consequentialist fairness preferences therefore concerns the dynamic inconsistency of these preferences. A decision maker may evaluate some outcome differently ex-ante, before a procedurally fair process has been implemented, versus ex-post, after its implementation has led to some distribution of outcomes. This happens when the consideration of counterfactual outcomes is easy ex-ante but difficult ex-post. Machina's (1989) parental example illustrates the problem. A mother of two children, Abigail and Benjamin, has exactly one candy. Both children agree that the candy will be allocated by coin flip (fair process). After Abigail wins the candy, Benjamin complains that it is unfair that only Abigail receives a treat (unfair outcomes). He is unable to appropriately consider the counterfactual situation of himself winning the candy. Similar problems arise in all situations where scarce resources are allocated by a fair process, but lead to unfair outcomes: agents obtaining the unfavorable outcome may ex-post question the fairness or appropriateness of the process, despite their initial acceptance of it.

Issues relating to the dynamic consistency of process fairness, and more generally the role of ex-ante versus ex-post evaluations of fairness, have been discussed in a few theoretical contributions. Andreozzi et al. (2013) present a model of a decision maker who is concerned about the correlation of her own and another person's payoffs in the context of risky random allocations. Fudenberg and Levine (2012) show that process fairness preferences are incompatible with the independence axiom underlying expected utility representations, and Saito (2013) subsequently introduces an axiomatic nonEU model of process and outcome fairness. Chassang and Zehnder (2016) study incomplete contracts with an arbitrator who is concerned about ex-ante and ex-post fairness. Most closely related to the current study, Trautmann and Wakker (2010) present the dynamic inconsistency problem inherent to process fairness.

While there is clear empirical evidence that process fairness plays an important role in many societal settings (e.g. Brock et al. 2013; Krawczyk 2010; Krawczyk and Le Lec 2010; Linde and Sonnemans 2015; Schildberg-Hörisch 2010 and references therein ${ }^{1}$ ), there is little evidence yet on the dynamic consistency of process fairness preferences (see Trautmann and Wakker 2010, section 4). Apart from our current results, the only paper directly assessing the dynamic consistency of process fairness preference is Andreoni et al. (2016). These authors investigate dynamic consistency of fairness preferences of an allocating third party (like the mother in Machina's example), and find evidence for dynamic inconsistency of fairness preferences. In Section 4 we discuss these findings in more detail in the context of our own results, which are based on the preferences of the agents who are themselves exposed to a random allocation of their outcomes (like Benjamin and Abigail in Machina's example). We believe that

\footnotetext{
${ }^{1}$ A slightly different approach concerns the opposite effect, namely the influence of fairness concerns on preferences for risky prospects (e.g., Rohde and Rohde 2011; Lahno and Serra-Garcia 2015; or Linde and Sonnemans 2012).
} 
establishing the prevalence of violations of dynamic inconsistency under process fairness preferences is of major importance for applications. If dynamic consistency cannot be empirically supported, preferences may typically revert to outcome fairness after procedures have been executed, with various implications for contracts and the feasibility of organizational outcomes.

In many settings, process fairness to some extent depends on whether other agents subscribe to it as well. Consider Machina's example. If the mother is responsive to Benjamin's ex-post complaint, Abigail may feel that the procedure was not fair after all. Such a dependence on the preferences of the other agents does not apply to outcome fairness. In practical applications, it will typically be the case that agents will not know for sure whether others subscribe to process fairness, or to outcome fairness, or possibly are dynamically inconsistent. Consider for example a fair promotion process. An unsuccessful candidate may afterwards try to delay or even prevent the promotion of the selected candidate by legal means, despite his initial agreement with the procedures for assessment. But then the process was not balanced after all. For a candidate who consistently subscribes to process fairness, the outcomes are either the other candidate getting promotion, or his own selection for promotion being contested. Because of this additional problem for process fairness (which is absent for outcome fairness), we study whether people consistently hold the process fairness view in situations where there is some dependence on other people behaving in accord with process fairness as well. That is, we implement a demanding test of process fairness.

The fairness of an allocation process can be established unambiguously in an experimental setting. However, in practical situations, the process may be ambiguous. To probe the robustness of process fairness when the process is ambiguous, we study dynamic consistency of fairness preferences both if the random distribution is known to the decision makers ("risky process") and if it is unknown ("ambiguous process"). Because decision makers often hold pessimistic attitudes toward ambiguous random processes (Ellsberg 1961; Trautmann and van de Kuilen 2015; Viscusi and Magat 1992), ambiguity may lead to a biased perception of the fairness of the allocation process. That is, the decision makers may put too much weight on the poor allocation outcomes for themselves and too little weight on the good ones, leading to a breakdown of process fairness. Ambiguity may also make it easier to justify a switch towards the outcome focus after observing a bad outcome ex-post, leading to more dynamic inconsistency.

We study dynamic consistency of social preferences in a simple 2-player random allocation game (described in detail in Section 2.1). In the presence of uncertainty about the selected allocation, players are asked whether they accept or reject a highly disadvantageous and a highly advantageous allocation, respectively. Allocations are only implemented if both players accept it. Otherwise an outcome-fair allocation with a low payoff for both players is implemented. ${ }^{2}$ In a $2 \times 2$ between-subjects design, we vary the nature of the random process that determines whether the advantageous or the disadvantageous allocation is proposed (risky vs. ambiguous), and the point in time when players are asked to make their decision, relative to the moment that the uncertainty concerning the proposed allocation is resolved (ex-ante vs. ex-post). We

\footnotetext{
${ }^{2}$ Importantly, this allocation is strictly better than the disadvantageous allocation from the perspective of outcome fairness, and from a purely selfish perspective concerned only about own payoffs.
} 
observe that a substantial share of the participants accept the disadvantageous allocation ex-post, i.e., after the resolution of uncertainty (30\% in the risky environment and $24 \%$ in the ambiguous environment). When decisions are made from the ex-ante rather than the ex-post perspective, the percentage of accepted disadvantageous allocations is somewhat higher (37\% in the risky environment and $46 \%$ in the ambiguous environment). We also observe direct evidence for dynamic inconsistency in the ex-ante condition: when offered the chance to reconsider their ex-ante decisions, about $20 \%$ of the participants change their decision and do so significantly more often in the direction predicted by outcome fairness or selfishness.

Thus, even in an environment that is arguably unfavorable to process fairness, a significant share of decision makers behave according to process fairness in both exante and ex-post decisions, rejecting consequentialism. However, there is some evidence for dynamic inconsistency, with outcomes being more salient after the resolution of uncertainty. Institutional arrangements based on procedural fairness thus need to be on guard against a potential "unraveling" of process fairness as outcomes are determined and communicated to those exposed to the allocation process.

\section{The random allocation game}

\subsection{Random allocation game and fairness preference}

We study the behavior of two players, A and B, in the random allocation game shown in Fig. 1 (the figure and description in this section concern the condition with a known probability, risky allocation process). An equal chance random draw determines the state of the world, UP or DOWN. If UP obtains, the allocation mechanism proposes a highly unfair allocation to the advantage of A, namely (A: €9, B: €1). This allocation is only implemented if both players accept it. If at least one player rejects the allocation, the fair allocation (A: $€ 2, \mathrm{~B}: € 2$ ) will be implemented. Note that player B would prefer the latter allocation both if she is concerned about outcome fairness, and if she is only concerned about her own payoffs. If DOWN obtains from the random draw, the opposite situation will obtain, with the allocation (A: €1, B: €9), advantageous to $B$, implemented if both players agree, and the symmetric allocation (A: €2, B: €2) otherwise. $^{3}$

We implement the decision to accept or reject each of the two unfair allocations under two conditions that vary the point in time when the player is asked to make the decision, relative to the moment the random draw is realized. In condition Ex-Ante, both players had to decide for each allocation whether they wanted to accept or reject it, before they knew the outcome of the random draw. In condition Ex-Post, both players were asked to decide after the random draw whether they wanted to accept or reject the allocation that was actually selected by the random mechanism.

\footnotetext{
${ }^{3}$ Note that this calibration of the payoffs does not allow us to disentangle preferences for outcome fairness from own-payoff maximization. Our goal is to identify process fairness in the presence of these potential alternative considerations.
} 


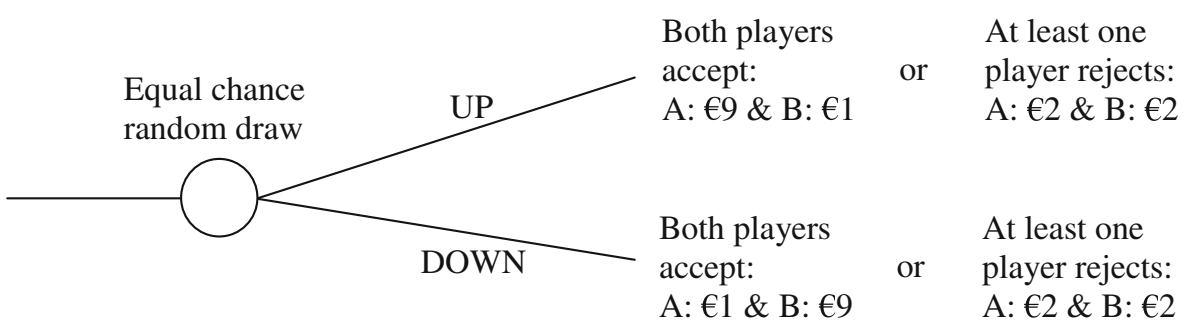

Fig. 1 Random allocation game

From the perspective of a person who only considers her own payoffs or cares about outcome fairness, the equal payoff allocation is preferred in the situation where the unfair allocation is to her own disadvantage. In the situation where the unfair allocation is to her advantage, a selfish person prefers the unfair allocation. For a person who cares about outcome fairness this is also true if her dislike of advantageous inequality is not too strong. In that case the benefit of receiving $€ 9$ rather than $€ 2$ will still lead her to accept the allocation. That implies that with selfish or outcome-fairness oriented players, in both branches of the decision tree at least one player rejects the unfair offer, and the payoffs will therefore be (A: $€ 2$, B: $€ 2)$ in both states of the world. In contrast, a person who considers the process fairness perspective takes the full decision tree into account in her decision even after the uncertainty has been resolved. Because the random device allocates the two equally unfair allocations symmetrically between the two players, it may be considered fair: if both players accept both unfair allocations, the expected payoffs would equal $€ 5$ for both players. Equality of expected payoffs has been suggested as a tractable criterion for process fairness. ${ }^{4}$

Note that the assumption that both people subscribe to process fairness is crucial. If player B rejects the disadvantageously unfair allocation in UP, while player A accepts the disadvantageously unfair allocation in DOWN, the expected allocation equals (A: $€ 1.5, \mathrm{~B}: € 5.5)$, which is not fair. Although it can be argued that the random process is still fair, behavior by the other player affects the distribution of both expected and realized payoffs and might therefore make the implementation of process fairness more difficult. Moreover, risk aversion would make it harder to implement process fairness because the outcome view offers a sure $€ 2$, while the process view offers an uncertain expected payoff of $€ 5$. We think that these factors are relevant in many settings, including the simple parental example. Our design allows us to gauge the relevance of process fairness in such a situation, thus providing a demanding empirical test of the robustness of the procedural fairness view.

An important consequence of process fairness is the possibility to implement socially beneficial though unequal allocations (in terms of outcomes). That is, process fairness is attractive to people who have a preference for maximizing social welfare. Indeed, in the current setup the procedurally fair but unequal allocation is preferable from the process fairness view only if it leads to a higher sum of the individual payoffs. People with a preference for social welfare maximization, e.g. as proposed by Charness and Rabin (2002), should therefore also consistently accept all unfair allocations in both the ex-ante and ex-post condition. ${ }^{5}$

\footnotetext{
${ }^{4}$ E.g., Bolton et al. (2005); Trautmann (2009); Krawczyk (2011).

${ }^{5}$ Bolton et al. (2005) report that people accept disadvantageously unfair but socially optimal allocations under fair processes, but not under unfair processes.
} 


\subsection{Random allocation game and dynamic consistency}

We compare acceptance rates for unfair allocations in the Ex-Ante with those in the ExPost condition to evaluate dynamic consistency in a between-subjects design. If process fairness is consistently applied by people in a population, we would observe a similar degree of preference for accepting an unfair allocation ex-ante and ex-post. To also obtain some insights on behavior at the within-person level, we asked subjects who made their decision at the ex-ante position to confirm (or change) their decision after the random draw had been conducted (see screen shot in the appendix). If process evaluations are more intuitive or convincing before the resolution of uncertainty than afterwards, people may reconsider their choices when given the opportunity to do so. Note that consistency in the between-subjects design does not necessarily imply consistency in the within-subjects design, or vice versa. While on the one hand it may be easier to transfer the ex-ante view to the ex-post decision in the within-person setting, emotional reactions may also evolve differently in a dynamic choice setting after observing the disadvantageous state of the world obtained (e.g., Hopfensitz and van Winden 2008). This may work against consistency in the within-person design.

\subsection{Ambiguous allocation process}

To study the effect of a potentially more realistic ambiguous allocation process we implement the above described decision situations in an additional condition using an ambiguous lottery to determine the allocation, resulting in a $2 \times 2$ (Ambiguous vs. Risky x Ex-Post vs. Ex-Ante) between-subjects factorial design. In contrast to the risky-process conditions in which there is a known 50-50 chance for allocations UP or DOWN, in the Ambiguous conditions the allocation is determined by the color of a card drawn from a deck of ten playing cards. Each card is either red or black, but the proportion of colors in the deck is unknown to the players; no further information about the distribution of cards is provided. That is, the allocation mechanism can potentially be very unbalanced. Experiments on ambiguity attitudes often use this setup, and typically find that people hold pessimistic attitudes toward these types of lotteries (e.g., Trautmann and van de Kuilen 2015).

Apart from the variation in the allocation process, the ambiguous conditions were exactly identical to the conditions with a known-risk process. Instructions for all four conditions are given in the appendix.

\subsection{Lab details}

In total, 202 subjects participated in the computerized laboratory experiment (Fischbacher 2007), 46 in the Ex-Ante-Risky condition, 54 in the Ex-Post-Risky condition, 52 in the Ex-Ante-Ambiguous condition, and 50 in the Ex-PostAmbiguous condition. The random allocation game followed on an unrelated individual decision making task which was identical for all subjects; subjects had not received any feedback on the outcome of the first task when they made their decisions in the random allocation game. All instructions were distributed on paper and read aloud. The 
random allocation game task took about 10 minutes, while the whole experiment took about 30 minutes. At the end of the experiment one task was randomly selected for payment, subjects were paid in private, and then dismissed from the laboratory.

\section{Results}

\subsection{Between-subjects comparison of ex-ante and ex-post prevalence of process fairness}

Risky process Results for the Risky condition are shown in Table 1. As the second column of the table shows, $91.3 \%$ of the subjects accept the advantageous allocation when asked to make their decisions before the resolution of uncertainty $(N=46)$. Moreover, $37.0 \%$ of the subjects accept the disadvantageous allocation, rejecting outcome fairness from the ex-ante perspective. In the expost condition (fourth column in Table 1), i.e., when subjects were asked to indicate their decision after the resolution of uncertainty, subjects made only one decision depending on the allocation that was selected. In this case, $100 \%$ of the subjects accept the advantageous allocation $(N=27)$, while $29.6 \%$ accept the disadvantageous one $(N=27)$, rejecting outcome fairness from the ex-post perspective. The share of people accepting the disadvantageous allocation is not significantly different between the ex-ante and the ex-post condition $(p=.614$, Fisher exact test). ${ }^{6}$

Ambiguous process Results for the ambiguous process are shown in Table 2. As indicated in the second column of the table, $98.1 \%$ of the subjects accept the advantageous allocation in the ex-ante condition $(N=52)$, while $46.2 \%$ accept the disadvantageous allocation, rejecting outcome fairness from the ex-ante perspective. In the ex-post condition, subjects made only one decision, depending on the allocation that was selected. The fourth column in Table 2 shows that $100 \%$ of the subjects accept the advantageous allocation $(N=25)$, while $24 \%$ accept the disadvantageous allocation $(N=25)$, rejecting outcome fairness from the ex-post perspective. The share of people accepting the disadvantageous allocation is marginally different between the ex-ante and the ex-post situations ( $p=.082$, Fisher exact test).

Comparing the risky and the ambiguous conditions, we find no significant differences in the prevalence of process fairness. Pooling the data of both conditions, there is an overall marginally significant reduction of the number of people subscribing to process fairness from $41.8 \%$ in the Ex-Ante to $26.9 \%$ in the Ex-Post conditions ( $p=.078$, Fisher exact test), indicating dynamic inconsistency.

Result 1: A significant share of decision makers reject outcome fairness (as well as selfish profit maximization) from both the ex-ante and the ex-post perspective. The share is somewhat larger for ex-ante evaluations, suggesting larger relevance of process fairness when outcomes are not yet determined.

\footnotetext{
${ }^{6}$ All tests reported in the paper are two-sided tests.
} 
Table 1 Choices in Risky Process Condition

\begin{tabular}{lccc}
\hline & Ex-ante & & Ex-post \\
\cline { 2 - 3 } & Initial choice & Reconsider after uncertainty resolved & \\
\hline Advantageous allocation & $91.3 \%$ accept & $0 \%$ & $100 \%$ accept \\
& $8.7 \%$ reject & $75.0 \%$ & $0 \%$ reject \\
Disadvantageous allocation & $37.0 \%$ accept & $55.6 \%$ & $29.6 \%$ accept \\
& $63.0 \%$ reject & $14.3 \%$ & $71.4 \%$ reject \\
\hline
\end{tabular}

a conditional probabilities: conditional on initial choice indicated in the same line

\subsection{Within-subjects evidence for dynamic inconsistency}

Risky process In the Ex-Ante conditions, after making their decisions the decision makers were given a chance to reconsider their choice after learning about the actual outcome of the random process. We may expect that there are various psychological reasons not to change the decision ex-post, including a preference for consistency (e.g., Falk and Zimmermann 2016) or simple inertia (Samuelson and Zeckhauser 1988), even if the decision maker regrets her initial choice. Consistent with this view, we find only modest switching, with overall $21.7 \%$ of the decision makers reconsidering their initial choice; specifically, $13.0 \%$ change after an advantageous outcome obtained $(N=23)$, and $30.4 \%$ change after a disadvantageous outcome obtained $(N=23)$. However, we do find evidence that switching is non-random. After a good outcome, $0 \%$ of those who initially accepted the offer, but $75 \%$ of those who initially rejected the offer switch when given the opportunity ( $p<.001$, Fisher exact test). Similarly, after a bad outcome, $14.3 \%$ of those who initially rejected the offer, but $55.6 \%$ of those who initially accepted the offer switch when given the opportunity $(p=.066$, Fisher exact test). That is, those subjects who switch do so predominantly in the direction of outcome fairness or payoff maximization.

Ambiguous process Results are similar in the case of an ambiguous process. Overall we find that $15.4 \%$ of the decision makers reconsider their initial choice: $3.8 \%$ change after an advantageous outcome obtained $(N=26)$, and $26.9 \%$ change after a disadvantageous outcome obtained $(N=26)$. We find some evidence that switching is non-

Table 2 Choices in Ambiguous Process Condition

\begin{tabular}{lccc}
\hline & Ex-ante & & Ex-post \\
\cline { 2 - 4 } & Initial choice & Reconsider after uncertainty resolved $^{\mathrm{a}}$ & \\
\hline Advantageous allocation & $98.1 \%$ accept & $0 \%$ & $100 \%$ accept \\
& $1.9 \%$ reject & $100.0 \%$ & $0 \%$ reject \\
Disadvantageous allocation & $46.2 \%$ accept & $41.7 \%$ & $24.0 \%$ accept \\
& $53.8 \%$ reject & $14.3 \%$ & $76.0 \%$ reject \\
\hline
\end{tabular}

a conditional probabilities: conditional on initial choice indicated in the same line 
random. After a good outcome, $0 \%$ of those who initially accepted the offer, but $100 \%$ of those who initially rejected the offer switch when given the opportunity ( $p=.038$, Fisher exact test). After a bad outcome, $14.3 \%$ of those who initially rejected the offer, and $41.7 \%$ of those who initially accepted the offer switch when given the opportunity, a non-significant difference $(p=.190$, Fisher exact test). Thus, also for the ambiguous allocation process subjects who switch do so predominantly in the direction of outcome fairness or payoff maximization.

Result 2: When given the opportunity to reconsider their ex-ante choice, about $20 \%$ of subjects change their choice. People are more likely to switch after a negative outcome, and more likely to switch in the direction of outcome-fairness or selfishness, implying dynamically inconsistent process-fairness preferences.

\section{Conclusion}

This paper studies the prevalence and dynamic consistency of process fairness preferences in a decision situation in which the decision maker could not be sure that the other player subscribes to process fairness. A failure of the other player to do so would render the process unfair to the decision maker. Moreover, both outcome fairness and selfish payoff maximization work against process fairness in the current setting. Despite this strong demand on process fairness, we find that about $42 \%$ of the participants behave according to process fairness when making their choices before the resolution of uncertainty. About $27 \%$ behave according to process fairness when making their choices after the resolution of uncertainty. That is, process fairness seems to be somewhat more plausible when outcomes are not yet determined. Still, a significant share of the decision makers reject consequentialism in both ex-ante and ex-post conditions.

We also find some direct evidence for dynamic inconsistency. Few people reconsider their choices after the uncertainty has been resolved. However, if they change their decision, they do so in the direction dictated by a focus on outcomes, showing the relevance of consequentialism. Interestingly, all results are very consistent irrespective of whether the random allocation is based on a known-risk process, as in many experiments, or based on an ambiguous process, as in most practical applications.

Comparison of our results with recent work by Andreoni et al. (2016) provides some interesting insights. These authors focus on the behavior of a person who decides on the random allocation of a potential payment to either of two recipient families in a developing country. Ex-ante and ex-post conditions differ with regard to the winning chances of the two families after an initial random draw. Thus, while our study is concerned with decision makers who are themselves exposed to a random allocation of their payoffs, Andreoni et al. (2016) consider the case of a neutral, financially unmotivated, third party. Despite the substantial differences in the design of the two studies, results are mostly consistent. Similar to our results, Andreoni et al. find evidence for process fairness in both the ex-ante and in the ex-post framing. However, differences are much more pronounced, with a very strong tendency towards the fair process ex- 
ante, and a much weaker incidence of process evaluations ex-post. Consequently, these authors also find more pronounced evidence of dynamic inconsistency. The higher degree of dynamic consistency in our study might derive from the fact that we set a high threshold for process fairness, skewing the design against the process view. The $42 \%$ of the participants that subscribe to the process fairness view ex-ante despite the high threshold we implement may also be the ones who stick to it most resolutely. Alternatively, there may be differences in dynamic consistency between the fairness perception of the affected agents and an allocating neutral third party. These are fruitful directions for future investigations of dynamic fairness issues.

Overall thus, there is clear evidence that a significant share of people consistently subscribe to process fairness even in a situation where the odds are stacked against it. However, the finding that consequentialism has some effect on these decision makers as well suggests that institutional arrangements based on fair process evaluations need to make sure that process fairness evaluations do not "unravel" as outcomes are determined. In the setting of our initial example, Mom should stand firm and reject Benjamin's request for reconsideration.

Acknowledgements We are grateful to the editor, the anonymous reviewer, and to the participants at the 2015 HEC Paris Decision Making workshop for very helpful suggestions.

\section{Appendix}

\section{A. Screen shot: Confirmation of preference ex-post}

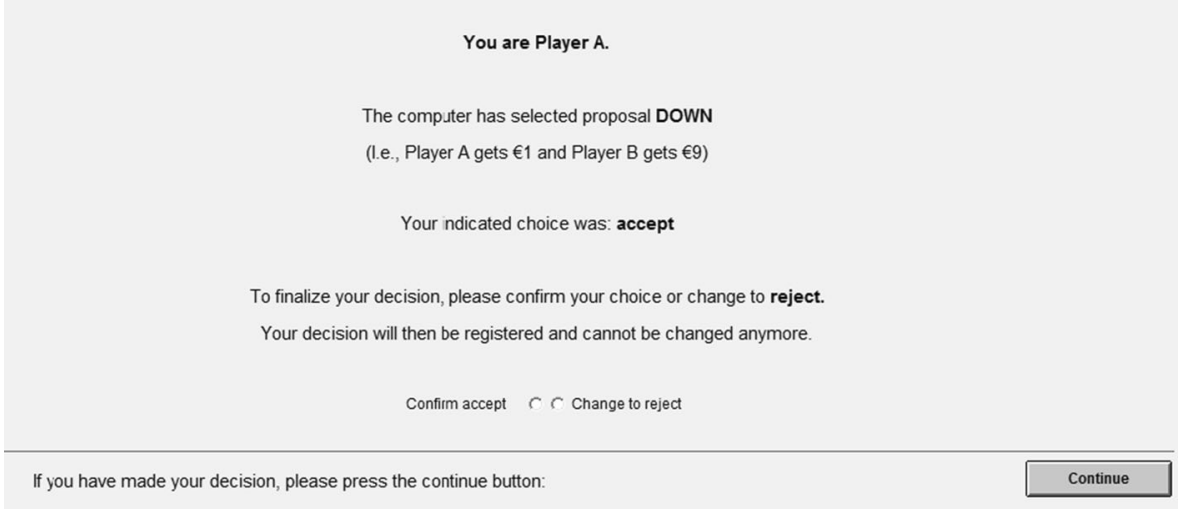

\section{B. Instructions Ex-Ante, Risk}

You interact with a randomly selected other person in the room. The interaction is anonymous, thus you will not learn the identity of the other person. In the game, one of you will be person A and the other one will be person B. This information will be shown on the screen before you make any choices. 
You interact in the following game:

The computer makes an equal chance (50-50) random draw that determines a proposal for the division of 10 Euros between both players as follows:

- If the computer chooses UP, the proposal will be "9 Euros for person A and 1 Euro for person B"

- If the computer chooses DOWN, the proposal will be "1 $\mathbf{1}$ Euro for person $A$ and 9 Euros for person B"

Before knowing whether the computer chooses UP or DOWN, you and the other person have to decide simultaneously whether to accept or reject each of the two proposals. Then, the computer determines which proposal will be implemented. If both of you indicated that you want to accept the selected proposal, the payoffs will be implemented. If at least one of you indicated that he/she wants to reject this proposal, the proposal will not be implemented and the payoff will instead be " 2 Euros for person A and 2 Euros for person B." See the graphical illustration.

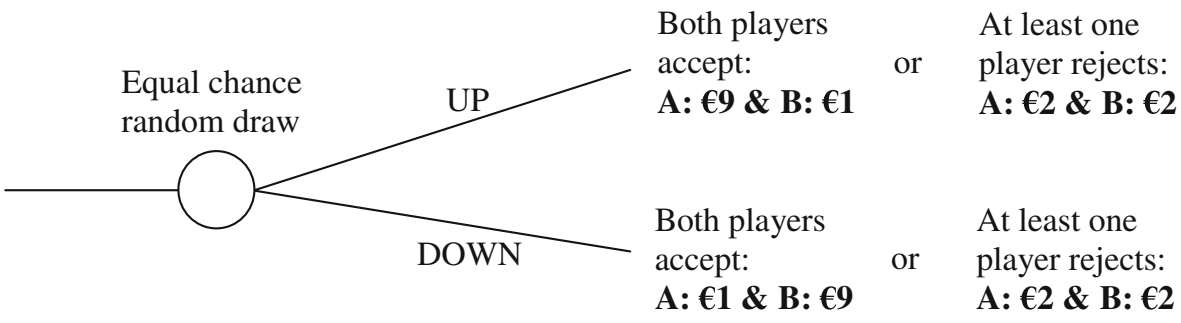

Please raise your hand if you need further explanation from the experimenter.

\section{Instructions Ex-Post, Risk}

You interact with a randomly selected other person in the room. The interaction is anonymous, thus you will not learn the identity of the other person. In the game, one of you will be person $\mathrm{A}$ and the other one will be person $\mathrm{B}$. This information will be shown on the screen before you make any choices.

You interact in the following game:

The computer makes an equal chance (50-50) random draw that determines a proposal for the division of 10 Euros between both players as follows:

- If the computer chooses UP, the proposal will be "9 Euros for person A and 1 Euro for person B"

- If the computer chooses DOWN, the proposal will be "1 Euro for person $\mathbf{A}$ and 9 Euros for person B"

After learning whether the computer chooses UP or DOWN, you and the other person have to decide simultaneously whether to accept or reject the selected proposal. If both of you indicate that you want to accept the selected proposal, the payoffs will be 
implemented. If at least one of you indicates that he/she wants to reject this proposal, the proposal will not be implemented and the payoff will instead be " 2 Euros for person A and 2 Euros for person B." See the graphical illustration.

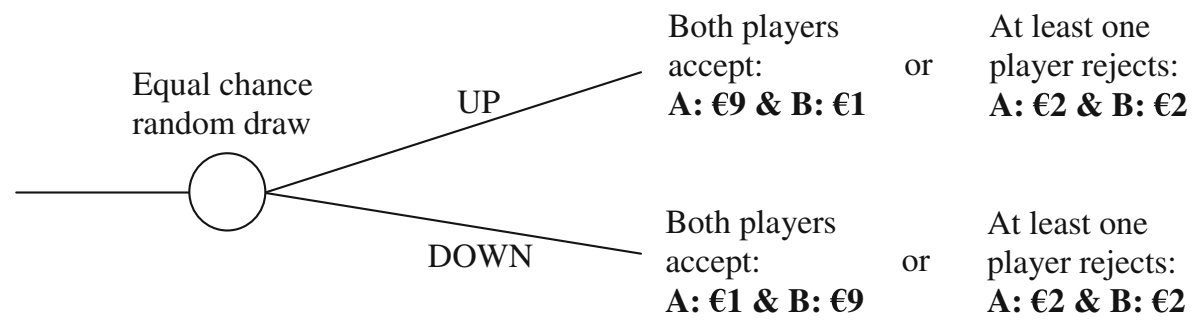

Please raise your hand if you need further explanation from the experimenter.

\section{Instructions Ex-Ante, Ambiguity}

You interact with a randomly selected other person in the room. The interaction is anonymous, thus you will not learn the identity of the other person. In the game, one of you will be person A and the other one will be person $\mathrm{B}$. This information will be shown on the screen before you make any choices.

You interact in the following game:

The experimenter will randomly draw a card from a deck of 10 cards, all of which are either red or black. Any combination of red and black cards is possible, but is unknown to you. The color of the card determines a proposal for the division of 10 Euros between both players as follows:

- If the card is RED, the proposal will be " 9 Euros for person $A$ and 1 Euro for person B"

- If the card is BLACK, the proposal will be "1 Euro for person A and 9 Euros for person B"

Before knowing whether the card is RED or BLACK, you and the other person have to decide simultaneously whether to accept or reject each of the two proposals. Then, the experimenter will draw the card from the bag to determine which proposal will be selected. If both of you indicated that you want to accept the selected proposal, the payoffs will be implemented. If at least one of you indicated that he/she wants to reject this proposal, the proposal will not be implemented and the payoff will instead be " 2 Euros for person A and 2 Euros for person B." See the graphical illustration.

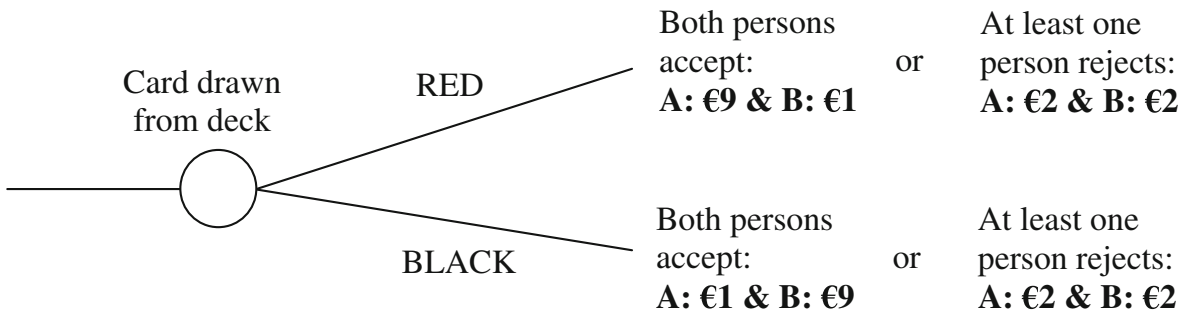


Please raise your hand if you need further explanation from the experimenter.

\section{E. Instructions Ex-Post, Ambiguity}

You interact with a randomly selected other person in the room. The interaction is anonymous, thus you will not learn the identity of the other person. In the game, one of you will be person A and the other one will be person $\mathrm{B}$. This information will be shown on the screen before you make any choices.

You interact in the following game:

The experimenter will randomly draw a card from a deck of 10 cards, all either red or black. Any combination of red and black cards is possible, but is unknown to you. The color of the card determines a proposal for the division of 10 Euros between both players as follows:

- If the card is RED, the proposal will be "9 Euros for person $A$ and 1 Euro for person B"

- If the card is BLACK, the proposal will be "1 Euro for person $A$ and 9 Euros for person B"

After learning whether the card is RED or BLACK, you and the other person have to decide simultaneously whether to accept or reject the selected proposal. If both of you indicate that you want to accept the selected proposal, the payoffs will be implemented. If at least one of you indicates that he/she wants to reject the proposal, the proposal will not be implemented and the payoff will instead be " 2 Euros for person A and 2 Euros for person B." See the graphical illustration.

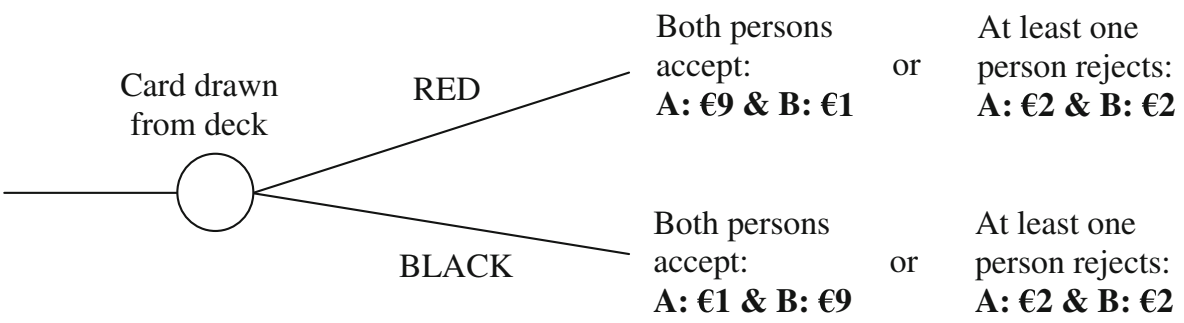

Please raise your hand if you need further explanation from the experimenter.

Open Access This article is distributed under the terms of the Creative Commons Attribution 4.0 International License (http://creativecommons.org/licenses/by/4.0/), which permits unrestricted use, distribution, and reproduction in any medium, provided you give appropriate credit to the original author(s) and the source, provide a link to the Creative Commons license, and indicate if changes were made.

\section{References}

Andreoni, J., Aydin, D., Barton, B., Bernheim, B.D., \& Naecker, J. (2016). When fair isn’t fair: sophisticated time inconsistency in social preferences. Working paper. 
Andreozzi, L., Ploner, M., \& Soraperra, I. (2013). Justice among strangers: on altruism, inequality aversion and fairness. Working paper.

Bolton, G. E., Brandts, J., \& Ockenfels, A. (2005). Fair procedures: evidence from games involving lotteries. Economic Journal, 115, 1054-1076.

Brock, J. M., Lange, A., \& Ozbay, E. (2013). Dictating the risk: experimental evidence on giving in risky environments. American Economic Review, 103, 415-437.

Charness, G., \& Rabin, M. (2002). Understanding social preferences with simple tests. Quarterly Journal of Economics, 117, 817-869.

Chassang, S., \& Zehnder, C. (2016). Rewards and punishments: informal contracting through social preferences. Theoretical Economics, 11(3), 1145-1179.

Ellsberg, D. (1961). Risk, ambiguity and the Savage axioms. Quarterly Journal of Economics, 75, 643-669.

Falk, A., \& Zimmermann, F. (2016). Consistency as a signal of skills. Management Science. doi:10.1287 /mnsc.2016.2459.

Fischbacher, U. (2007). Z-tree: Zurich toolbox for ready-made economic experiments. Experimental Economics, 10, 171-178.

Fudenberg, D., \& Levine, D. (2012). Fairness, risk preferences, and independence: impossibility theorems. Journal of Economic Behavior and Organization, 81, 606-612.

Hopfensitz, A., \& van Winden, F. (2008). Dynamic choice, independence, and emotions. Theory and Decision, 64, 249-300.

Krawczyk, M. (2010). A glimpse through the veil of ignorance: equality of opportunity and support for redistribution. Journal of Public Economics, 94, 131-141.

Krawczyk, M. (2011). A model of procedural and distributive fairness. Theory and Decision, 70, 111-128.

Krawczyk, M., \& Le Lec, F. (2010). 'Give me a chance!' an experiment in social decision under risk. Experimental Economics, 13, 500-511.

Lahno, A., \& Serra-Garcia, M. (2015). Peer effects in risk taking: envy or conformity? Journal of Risk and Uncertainty, 50, 73-95.

Linde, J., \& Sonnemans, J. (2012). Social comparison and risky choices. Journal of Risk and Uncertainty, 44, 45-72.

Linde, J., \& Sonnemans, J. (2015). Decisions under risk in a social and individual context: the limits of social preferences? Journal of Behavioral and Experimental Economics, 56, 62-71.

Machina, M. J. (1989). Dynamic consistency and non-expected utility models of choice under uncertainty. Journal of Economic Literature, 27, 1622-1668.

Rohde, I. M. T., \& Rohde, K. I. M. (2011). Risk attitudes in a social context. Journal of Risk and Uncertainty, 43, 205-225.

Saito, K. (2013). Social preferences under risk: equality of opportunity versus equality of outcomes. American Economic Review, 103, 3084-3101.

Samuelson, W., \& Zeckhauser, R. J. (1988). Status quo bias in decision making. Journal of Risk and Uncertainty, 1, 7-59.

Schildberg-Hörisch, H. (2010). Is the veil of ignorance only a concept about risk? An experiment. Journal of Public Economics, 94, 1062-1066.

Trautmann, S. T. (2009). A tractable model of process fairness under risk. Journal of Economic Psychology, 30, 803-813.

Trautmann, S.T., \& van de Kuilen, G. (2015). Ambiguity attitudes. In G. Keren \& G. Wu (eds.), Blackwell Wiley Handbook of Judgment and Decision Making. Chapter 3, 89-116. Blackwell.

Trautmann, S. T,. \& Vieider, F.M. (2012). Social influences on risk attitudes: Applications in economics. In S. Roeser (ed.), Handbook of Risk Theory. Chapter 22, 575-600. Springer.

Trautmann, S. T., \& Wakker, P. P. (2010). Process fairness and dynamic consistency. Economics Letters, 109, $187-189$.

Viscusi, W. K., \& Magat, W. A. (1992). Bayesian decisions with ambiguous belief aversion. Journal of Risk and Uncertainty, 5, 371-387. 